\title{
Fully Endoscopic Lumbar Laminectomy for Treatment of Large Dorsal Ligamentum Flavum Cyst and Severe Spinal Stenosis: A Technical note on a Case Report \\ Jian Shen*, MD, PhD
}

Department of Surgery, Mohawk Valley Orthopedics, Center for Spine Regeneration Surgery, New York, USA

\begin{abstract}
Ligamentum flavum cysts are very rare and thought to develop due to facet joint hypermobility. When they cause significant cord or nerve compression, surgical decompression and excision of the cyst is the gold standard of treatment. Here we present a fully endoscopic approach for lumbar laminectomy, bilateral decompression, and en bloc excision of a large dorsal ligamentum flavum cyst in a 69-year-old male with severe lumbar spinal stenosis and neurogenic claudication, under local anesthesia with conscious sedation. Advantages and disadvantages for this approach were discussed.
\end{abstract}

Keywords: Ligamentum flavum cyst; Fully endoscopic lumbar laminectomy; Bilateral decompression

\section{Introduction}

Traditional open and tubular approaches for treatment of central and lateral recess spinal stenosis involve laminotomy or laminectomy with removal of overgrown ligamentum flavum and a portion of the medial facet joints in order to decompress the central canal and lateral recess. Fully-endoscopic posterior lumbar laminectomy follows similar principle but is a more minimally invasive approach with unique advantages. This case presentation describes a step-by-step technique for fully-endoscopic lumbar laminectomy and its unique advantages to achieve bilateral decompression with unilateral approach and en bloc excision of a large dorsal ligmentum flavum cyst.

\section{Case Presentation}

\section{History and presentation}

This is a 69 -year-old male presented with progressively worsening chronic low back pain, bilateral dorsal foot numbness, and neurogenic claudication. His ODI score was $42 \%$. His LS spine MRI showed bilateral L5 spondylolysis, multi-level disc degeneration, diffuse spondylosis, worst at L4/L5, where a significant sized central dorsal epidural cyst, facet and ligamentum hypertrophy causing severe central canal stenosis (Figure 1). An EMG study showed bilateral L5 radiculopathy. He had extensive conservative treatments including physical therapy and epidural steroid injections but unfortunately without significant improvement.

\section{Operative procedure}

Under conscious sedation with local anesthesia, this patient underwent full endoscopic lumbar laminectomy (bilateral decompression with unilateral approach) and excision of the cyst, with the iLESSYS Delta endoscopic system (Joimax GmbH, Karlsruhe, Germany). This system has a $13-\mathrm{mm}$ outer diameter, and $10.2-\mathrm{mm}$ inner diameter canula, the endoscope has a $10-\mathrm{mm}$ outer diameter and a 6-mm working channel, and a 15-degree viewing angle. Under endoscopic view, decompression was achieved using the high-speed endoscopic drill, straight graspers were used to remove ligamentum flavum, and endoscopic Kerrison punches (both 40-degree angle and 90-degree angle) with $1.5-\mathrm{mm}$ and $3.0-\mathrm{mm}$ footprints were used to remove additional bone and ligament. Meticulous dissection of the interface between the cyst capsule and the dura was done with blunttipped nerve hook, with the help of contant fluid irrigation, and the cyst and its entire capsule were removed carefully. Hemostasis was achieved
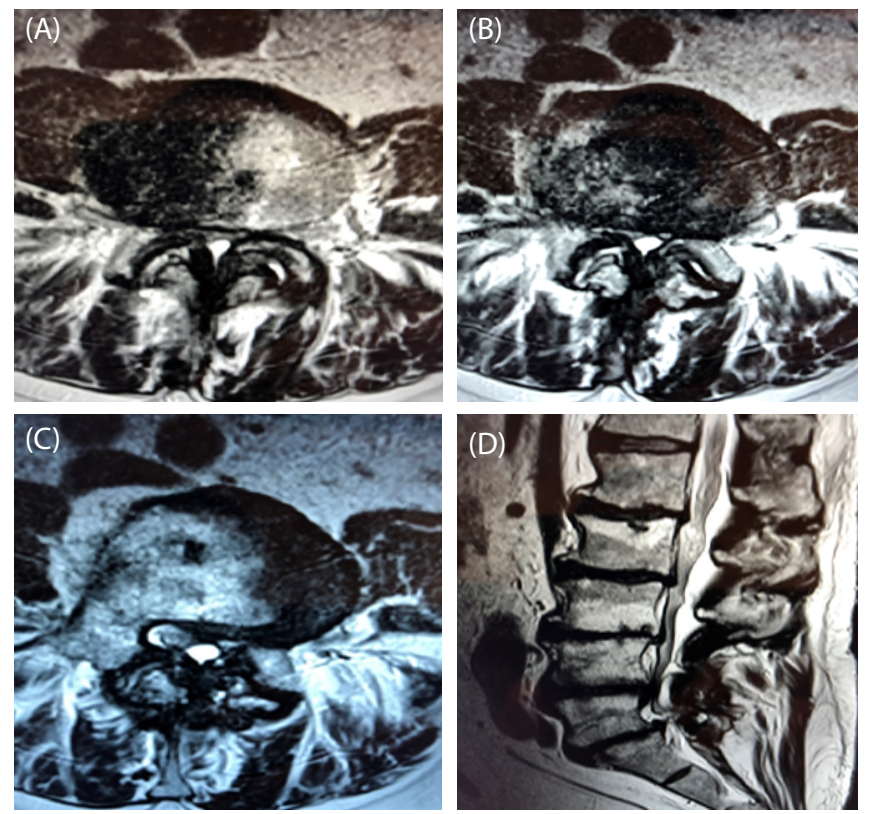

Figure 1: (A-D) T2-weighted axial and sagittal magnetic resonance images demonstrate a large L4/L5 ligamentum flavum cyst and severe thecal sac compression and spinal stenosis.

with a radiofrequency probe. At the end of decompression, the thecal sac and bilateral traversing nerve roots were seen well decompressed (Figure 2). The incision was closed in layers with absorbable sutures without a drain. The surgery took 96 minutes with minimal blood loss, without any complication. The patient was discharged home from recovery area. Pathology reports confirmed epidural synovial cyst.

*Corresponding author: Jian Shen, Spine Suregeon, Department of Surgery, Mohawk Valley Orthopedics, 5010 State Highway 30, Amsterdam, NY 12010; Center for Spine Regeneration Surgery, 215 East 77th Street, New York, NY 10075, USA, Tel: + 518842-2663; E-mail: james2173@yahoo.com

Received August 13, 2018; Accepted August 24, 2018; Published August 27, 2018

Citation: Shen J (2018) Fully Endoscopic Lumbar Laminectomy for Treatment of Large Dorsal Ligamentum Flavum Cyst and Severe Spinal Stenosis: A Technical note on a Case Report. J Spine S7: 011.doi:10.4172/2165-7939.S7-011

Copyright: (c) 2018 Shen J. This is an open-access article distributed under the terms of the Creative Commons Attribution License, which permits unrestricted use, distribution, and reproduction in any medium, provided the original author and source are credited. 

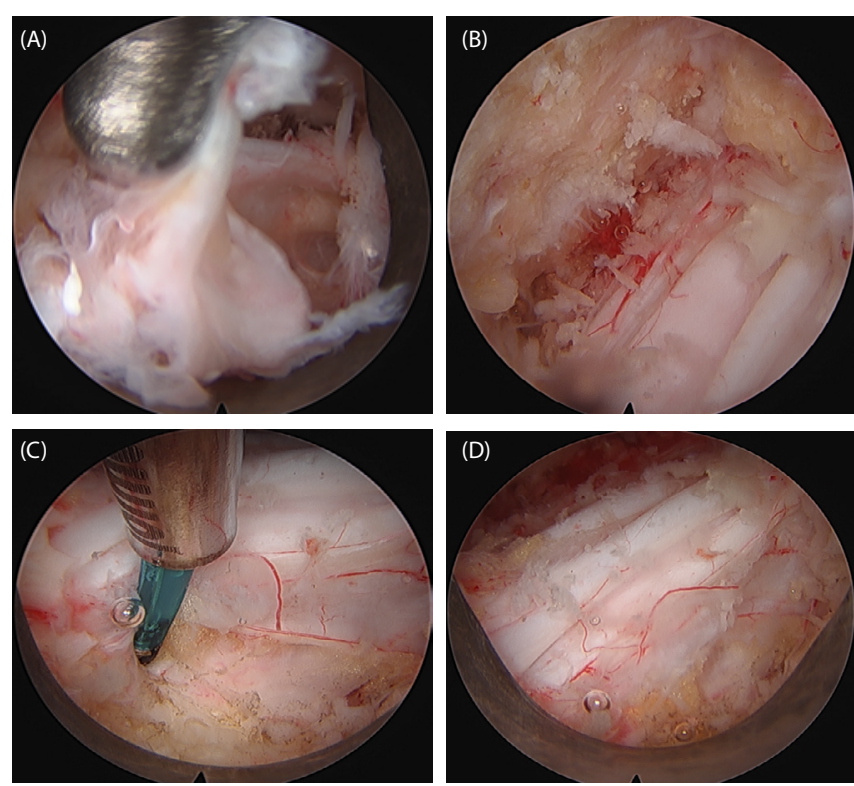

Figure 2: Intraoperative endoscopic photographs showing (A) Excision of the ligamentum flavum cyst capsule; (B) Contralateral lateral recess after decompression; (C) Ipsilateral side after decompression, with disc shown at 6 o'clock; (D) Decompressed thecal sac.

\section{Post-operative course}

Patient has been doing very well postoperatively, his prior low back pain and neurogenic claudication almost resolved immediately after surgery. One year after his endoscopic procedure, he continues to do well and his bilateral foot numbness has also resolved. His ODI score is $9 \%$.

\section{Discussion}

This is the first case report on treating large ligamentum flavum cyst/severe spinal stenosis with fully endoscopic approach. Ligamentum flavum cyst is considered as a category of juxtafacet cyst and is a rare cause of thecal sac/cord compression and spinal stenosis/radiculopathy. The pathogenesis of these cysts is not yet fully understood, but it has been proposed that continuous stress to this ligament due to minor repetitive trauma, such as in spondylolisthesis and degenerative disc disease, may lead to the development of cyst [1]. These cysts are unusual in cervical spine and even rarer in dorsal spine. Surgical decompression is the gold standard of treatment. This underwent a fully-endoscopic decompressive L4 laminectomy and en bloc excision of the ligamentum flavum cyst with complete resolution of his symptoms postoperatively. Because of better visualization and the constant irrigation of fluid, fully-endoscopic approach has unique advantages for decompression for the spinal canal and excision of the cyst.

The evolution of surgical techniques to decompress the central canal and lateral recess has been from traditional open laminectomy, to microscopic, micro endoscopic (MED), and fully-endoscopic techniques $[2,3]$. The goal of fully endoscopic laminectomy is the same as open decompression, yet it preserves midline structures and facet function/stability. Other advantages include better visualization, much less bleeding, less need for narcotic pain medications, quicker recovery. Most fully-endoscopic lumbar decompression cases can be done under local anesthesia with conscious sedation.

The disadvantages of fully-endoscopic laminectomy techniques described here include longer operative time, because it is a 'oneinstrument-at-a-time" technique [4].

\section{Conclusion}

In conclusion, repairing dural tear is very challenging because of the better visualization and the constant irrigation pushes the dura away from the bone and ligament being resected, the likelihood of dural tear is much lower in fully-endoscopic decompression surgeries. In case of dural tear, convert to tubular approach, primary suture repair under microscope is still believed to be the technique of choice.

\section{References}

1. Cakir E, Kuzeyli K, Usul H, Peksoylu B, Yazar U, et al. (2004) Ligamentum flavum cyst. J Clin Neurosci 11: 67-69.

2. Komp M, Hahn P, Oezdemir S, Giannakopoulos A, Heikenfeld R, et al. (2015) Bilateral spinal decompression of lumbar central stenosis with the full-endoscopic interlaminar versus microsurgical laminotomy technique: a prospective, randomized, controlled study. Pain Physician 18: 61-70.

3. Ralf Wagner AET, Guntram K, Iprenburg M (2018) Fully-endoscopic lumbar laminectomy for central and lateral recess stenosis: technical note. Interdisciplinary Neurosurgery 13: 6-9.

4. Tafazal SI, Sell PJ (2005) Incidental durotomy in lumbar spine surgery: incidence and management. Eur Spine J 14: 287-290. 